\title{
INVITED: In Quest of the Next Information Processing Substrate
}

\author{
Extended Abstract ${ }^{\dagger}$
}

\author{
Suman Datta, Alan Seabaugh, \\ Michael Niemier \\ University of Notre Dame \\ Notre Dame, IN 46556 \\ USA \\ \{sdatta, seabaugh.1, mniemier\} \\ @.nd.edu \\ Debdeep Jena, Grace Xing \\ Department of Electrical Engineering, \\ Cornell University \\ Ithaca, New York 14853 \\ USA \\ \{djena, grace.xing\}@cornell.edu
}

\author{
Arijit Raychowdhury \\ Georgia Institute of Technology \\ Atlanta, Georgia 30332 \\ USA \\ arijit.raychowdhury@ece.gatech.edu
}
H. -S. Philip Wong, Eric Pop
Stanford University
Stanford, CA 94305-9505
USA
\{hspwong, epop\}@stanford.edu

\author{
Darrell Schlom \\ Department of Materials Science and \\ Engineering, Cornell University \\ Ithaca, New York 14853 \\ USA \\ schlom@cornell.edu
}

\author{
Sumeet Gupta \\ The Pennsylvania State University \\ University Park, PA 16802 \\ USA \\ skgupta@psu.edu
}

\author{
Supratik Guha \\ University of Chicago \\ Chicago, IL 60637 \\ USA \\ guha@uchicago.edu
}

\begin{abstract}
Conventional CMOS scaling and the Moore's law have been the cornerstone of progress in computing hardware technology. However, with dimensional scaling expected to end soon, there is a pressing need to find the next information processing hardware that can continue to support the technology revolution. Will this hardware solution be an enhanced or an augmented version of MOSFET or a switch based on a radically new switching mechanism. Ultimately, do we require a complete deviation from the Boolean paradigm itself? In this invited paper, we will review some of the actively pursued future logic, merged logic-memory and related concepts.
\end{abstract}

Permission to make digital or hard copies of all or part of this work for personal or classroom use is granted without fee provided that copies are not made or distributed for profit or commercial advantage and that copies bear this notice and the full citation on the first page. Copyrights for components of this work owned by others than the author(s) must be honored. Abstracting with credit is permitted. To copy otherwise, or republish, to post on servers or to redistribute to lists, requires prior specific permission and/or a fee. Request permissions from Permissions@acm.org.

DAC '17, June 18-22, 2017, Austin, TX, USA

(C) 2017 Copyright is held by the owner/author(s). Publication rights

licensed to ACM. ACM 978-1-4503-4927-7/17/06 ..\$15.00

http://dx.doi.org/10.1145/3061639.3072953
While it remains unclear which of these options will eventually make it into commercial products, we will argue- based on lessons learnt from the past two decades of transistor development- that sustained and systematic research with careful benchmarking from future workload and applications viewpoint remains the key to success in the quest for the new information processing hardware.

\section{KEYWORDS}

FinFET; Quantum-Well FinFET; Tunnel FET; Phase-FET; NC FET; Oscillators; $\mathrm{VO}_{2}$

\section{ACM Reference format:}

S. Datta, A. Seabaugh, M. Niemier, A. Raychowdhury, D.G. Schlom, D. Jena, G. Xing, H.S-.P. Wong, E. Pop, S. Salahuddin, S. Gupta, and S. Guha. 2017. In Proceedings of ACM DAC conference, Austin, Texas USA, Jun 2017, 6 pages.

$10.1145 / 3061639.3072953$ 


\section{INTRODUCTION}

Technology scaling has led to an unprecedented level of integration with billions of high-speed nano-transistors on a single chip reducing the cost per function. On the device technology front, device engineers have achieved through continued scaling, new transistor breakthroughs, and introduced innovations at a rapid pace followed by successful launch of commercially successful products such as high performance microprocessors that presently power over a billion mobile smartphones, personal computers, and massive data centers.

We have achieved this by overcoming seemingly fundamental barriers to transistor scaling imposed by natural laws of physics allowing us to sustain an aggressive scaling roadmap in a persistent effort towards enhancing the energy efficiency of the transistors. We will outline this incredible journey that spans over last sixteen years of logic transistor research and development. We will argue that serendipity has always played a key role in shaping this remarkable path of transistor innovation. Recent innovations in silicon complementary metal oxide semiconductor (CMOS) transistors that have achieved successful commercial implementations include strained channel transistors, high-k metal gate transistors and non-planar Tri-gate transistors as illustrated in Fig. 1 [1-3].

The road ahead in our quest for the next transistor / switch as a hardware solution is fraught with complex challenges. New transistor architectures such as gate-all-around vertical and horizontal nanowires, new band engineered channels such as quantum-well FinFETs are being pursued as revolutionary transistors. At the same time, new switching mechanisms such as band-to-band tunneling and insulator-metal phase transitions are vigorously being explored to enable steep slope transistors. Some of these designs and architectures are even venturing into the concept of stabilizing the "negative capacitance" in a ferroelectric dielectric integrated within the gate stack to boost the performance of a conventional transistor. We will summarize the current stateof-the-art of these new transistors in the following sections.

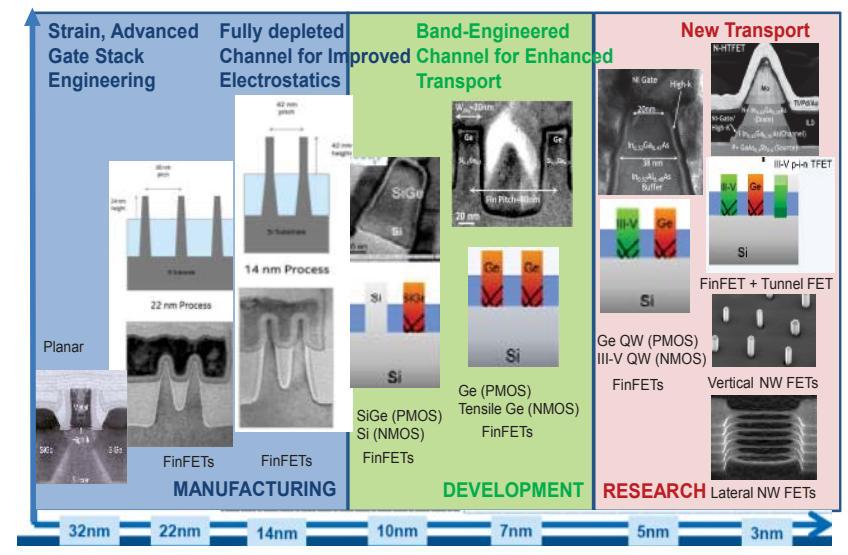

Figure 1: Logic transistor innovation and evolutionary roadmap
At this stage, it's not clear which of these options will find their way into commercial products. However, as history has proven before, the research will find its way into technologies in unexpected ways, provided we remain vigilant and committed.
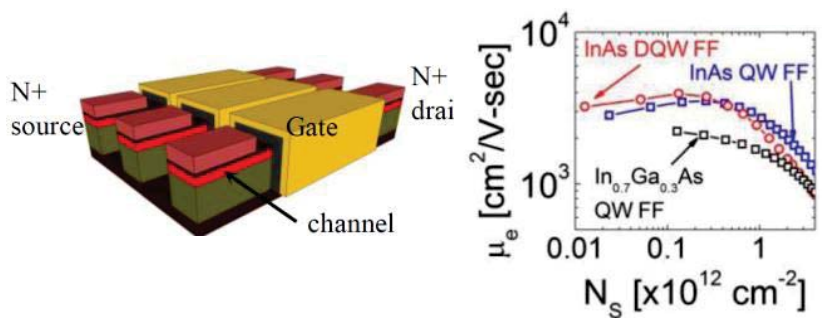

Figure 2: Quantum-well FinFET and measured room temperature effective mobilities.

\section{BOLTZMANN TRANSISTORS}

\subsection{Quantum-Well (QW) FinFETs}

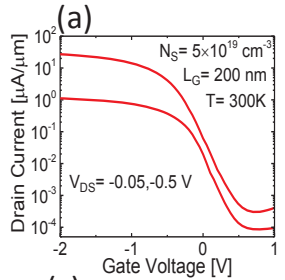

(c)

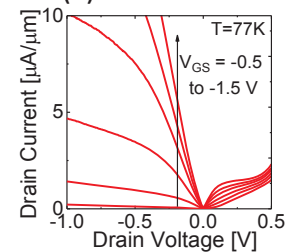

(e)

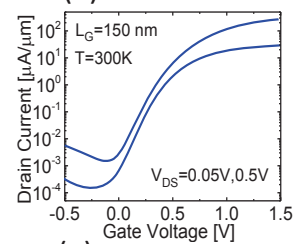

(g)

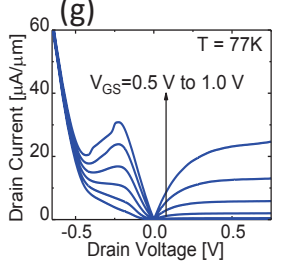

(b)

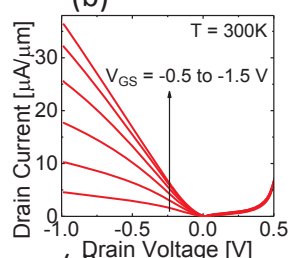

(d)

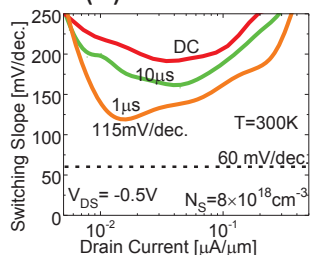

(f)

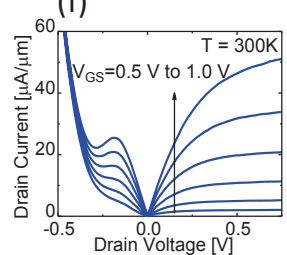

(h)

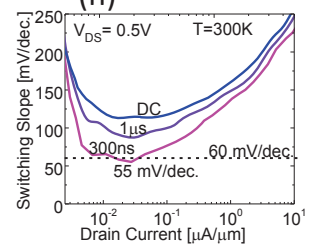

Figure 3: Transfer, output and switching characteristics of (a-d) PTFET and (e-h) NTFET [10]. All measurements are at $T=300 \mathrm{~K}$, except the additional $\mathrm{T}=77 \mathrm{~K}$ data in (c) and (g). NDR is visible in PTFET output characteristics at $T=77 \mathrm{~K}$, due to the suppression of trap response. Improvement in switching performance from pulsed mode measurements is evident from (d, h). 
Power constrained scaling of CMOS technology places several stringent requirements on the performance metrics of the underlying device, such as drive-current, leakage current, on-to- off state current ratio, subthreshold slope, Drain Induced Barrier Lowering (DIBL), all which have to be satisfied simultaneously. The key requirement however, is to reduce dynamic power dissipation which can be achieved by scaling down the supply voltage $(\mathrm{VDD})$ because power dissipation is proportional to the square of $\mathrm{V}_{\mathrm{DD}}$. At reduced $\mathrm{V}_{\mathrm{DD}}$, however, traditional silicon CMOS is limited by reduced drive currents. In this context, III-V compound semiconductor material systems are being actively researched because they afford competitive drive currents at lower supply voltages. The advantage primarily stems from the reduced effective mass in III-V materials which results in higher mobility. Recently, InGaAs and InAs heterostructure FinFETs have been demonstrated as an n-channel alternative to Si FinFET [4-6]. InAs single and dual Quantum Well (QW) FinFETs exhibit peak mobility $>3,000 \mathrm{~cm}^{2} / \mathrm{V}$-sec. Projected short-channel InAs QW FinFETs are expected to show $15 \%$ higher drive current with comparable short channel effects as Silicon FinFETs, making them a potential candidate for future transistors.

\subsection{Inter-Band Tunnel FETs (TFETs)}

In the future, even high mobility transistors will face supply voltage $V_{D D}$ scaling challenges as we near $500 \mathrm{mV}$ supply due to the fundamental $60 \mathrm{mV} /$ decade sub-threshold swing limitation in MOSFETs arising from the fundamental Boltzmann limit. Consequently, any further threshold voltage scaling leads to severe increase in static power dissipation. TFETs with gated band-toband tunneling at the source-channel junction [7] potentially leads to filtering of the high-energy tail of the Femi-Dirac distribution of electrons in the source of TFET. This band-pass filtering action at the source-channel junction leads to an effective "cooling" of the carrier population and results in sub- $k T / q$ or $s u b-60 \mathrm{mV} /$ decade of sub-threshold swing at room temperature. Thus, TFETs enable aggressive $V_{D D}$ scaling without degrading the ON-state performance of the transistors. Figure 3 shows the experimental transfer (IDS-VGS) and output (IDS-VDS) characteristics for the fabricated PTFET and NTFET. GaAs $0.35 \mathrm{Sb}_{0.65}$ channel PTFETs exhibit $\mathrm{ION}_{\mathrm{ON}}=30 \mu \mathrm{A} / \mu \mathrm{m}$ at $\mathrm{I}_{\mathrm{ON}} / \mathrm{I}_{\mathrm{OFF}}=10^{5}$. The PTFET output characteristics exhibit negative differential resistance (NDR) and saturation at low temperature as contribution from mid-gap $D_{i t}$ is suppressed. $\mathrm{In}_{0.65} \mathrm{Ga}_{0.35} \mathrm{As}$ channel NTFET shows $\mathrm{I}_{\mathrm{ON}}=275 \mu \mathrm{A} / \mu \mathrm{m}$ at $\mathrm{ION}_{\mathrm{ON}} / \mathrm{I}_{\mathrm{OFF}}=3 \times 10^{5}$. The switching slope $(\mathrm{SS})$ in the fabricated TFETs surpasses the Boltzmann limit of $60 \mathrm{mV} /$ decade at room temperature. This dilution of SS is a consequence of mid-gap $D_{\text {it }}$ with slow response time. To suppress the response of mid-gap $\mathrm{D}_{\mathrm{it}}$, we perform pulsed $\mathrm{I}_{\mathrm{DS}}-\mathrm{V}_{\mathrm{GS}}$ measurement on TFETs with the input gate-voltage $\mathrm{V}_{\mathrm{GS}}$ pulse having rise-times varying from $10 \mu \mathrm{s}$ down to $300 \mathrm{~ns}$ to assess actual switching performance. Fig. 3(d,h) shows the $\mathrm{SS}$ as a function of drain current for various gate pulse rise times. We achieve $\mathrm{SS}=55 \mathrm{mV} /$ decade for NTFET and $\mathrm{SS}=115 \mathrm{mV} /$ decade for PTFET at room temperature in pulsed mode measurements. Consequently, engineering high-quality scaled gate dielectrics and tunnel barriers in the As-Sb system is fundamental to the realization of high Ion with steep switching slope demonstration in TFETs.

\section{LANDAU TRANSISTORS}

While TFETs harness the alternate tunneling transport mechanism within conventional semiconductors, a new paradigm for enabling sub-kT/q switching devices exploits an entirely new class of materials with unique properties, enabled by novel materials physics, and unavailable in conventional semiconductors. Such phenomena include insulator-metal transitions, ferroelectricity, piezoelectricity; and in this context, their corresponding transistor implementations can be broadly classified as Landau transistors because of the similar double-well energy landscape associated with phase transitions described by the Landau theory [10]. Here, we will describe two Landau transistor concepts: The Phase-FET based on the phenomenon of abrupt insulator-metal transition (IMT), and the Negative Capacitance (NC) FET that harnesses negative capacitance in a composite ferroelectric dielectric gate stack, both of which are being actively pursued as steep-slope transistor options.

\subsection{Phase FETs}

Phase-FETs utilize the phenomenon of an abrupt insulatormetal transition (IMT) that arises from collective interactions in certain materials, and manifests itself as orders of magnitude change in resistivity. This change is induced due to a fundamental change in the band-structure, and the bandgap and subsequent amplification of the carrier concentration. For example, in the prototypical IMT material, vanadium dioxide $\left(\mathrm{VO}_{2}\right)$, an electrical stimulus applied to a two-terminal $\mathrm{VO}_{2}$ device collapses the $0.6 \mathrm{eV}$ bandgap in the insulating state, transforming $\mathrm{VO}_{2}$ into a metal (no bandgap) along with up to 4 orders of change in resistivity [11].

The concept of the phase-FET entails the integration of such IMT materials into the source of a conventional transistor (Fig. 4(a)) [12]. The gate-terminal voltage $V_{G S}$ modifies the current flowing through the series combination of the transistor channel and the IMT material, triggering the abrupt and reversible phase transition, and enabling the steep sub-kT/q switching characteristics. Further, the high-resistivity insulating state of the IMT material exponentially reduces the OFF-state leakage of the conventional FET while the orders of magnitude reduction in resistivity following transformation to the metallic state ensures negligible reduction in the ON state drive current of the FET enabling the Phase-FET to exhibit a higher ON-OFF ratio in comparison to the conventional FET; the higher ON-OFF ratio can be translated into higher ON current at matched OFF current of the MOSFET. Thus, such a transistor design seamlessly incorporates the abrupt switching phenomenon in IMT materials with the superior field-effect dynamics of conventional semiconductor based transistors to deliver improved performance. 

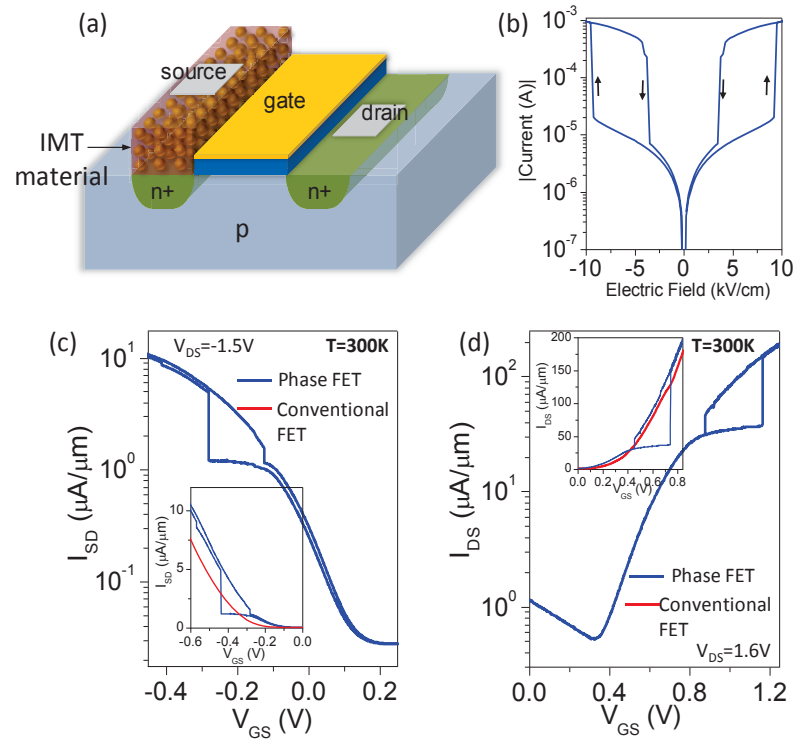

Figure 4: (a) Schematic of the Phase-FET consisting of the IMT material integrated with the source of a conventional transistor. (b) Typical I-V characteristics of a two-terminal $\mathrm{VO}_{2}$ device showing abrupt switching associated with the IMT. (c)(d) Transfer characteristics (IDS- $V_{G S}$ ) characteristics of the p-type and the n-type Phase FET, respectively. The conventional transistors for the p-type and n-type devices are p-Ge QW FinFET and n-In ${ }_{0.7} \mathrm{Ga}_{0.3} \mathrm{As}$ FinFET, respectively. Insets show the comparison of the Phase-FET with their respective conventional transistors at matched OFF current.

Figure $4(d)$ shows the transfer $\left(I_{D S}-V_{G S}\right)$ characteristics of a Phase-FET consisting of a n-type multi-channel $\mathrm{In}_{0.7} \mathrm{Ga}_{0.3} \mathrm{As} \mathrm{QW}$ FinFET in series with $\mathrm{VO}_{2}$. It can be observed from the inset of Fig. 4(d) that the Phase-FET delivers a $20 \%$ higher ON current in comparison to the conventional transistor at matched OFF state current. Further, the generality of the Phase-FET design makes it applicable to a p-type transistor as well. Fig. 4(c) shows a p-type Phase FET constructed using a Ge QW FinFET and $\mathrm{VO}_{2}$, exhibiting steep-switching characteristics, and $\sim 60 \%$ improvement in $\mathrm{ON}$ current in comparison to the conventional transistor at matched OFF-state current [12]. The steep-slope and higher ONOFF ratio enables VDD scaling, and subsequent reduction in dynamic power.

\subsection{Negative Capacitance (NC) FETs}

While the Phase-FET induces internal voltage amplification at the source side of a conventional MOSFET through the IMT phenomenon, the NC FET aims to exploit the concept of negative capacitance in a ferroelectric-dielectric gate stack to create an internal step-up transformer between the applied gate-voltage $\mathrm{V}_{\mathrm{GS}}$ and the surface potential $\Psi$ s. Negative capacitance, as predicted by the Landau mean-field theory, is an unstable region in the polarization (P) versus electric-field (E) characteristics of a ferroelectric where $\mathrm{dP} / \mathrm{dE}<0$. Salahuddin et al. [13] proposed that such an unstable NC region could be stabilized using a dielectric capacitance in series with the ferroelectric. The series combination of a positive capacitance (dielectric and semiconductor band bending) and an effective negative capacitance (arising from the ferroelectric) in the gate stack would amplify the internal node voltage $\Psi$ s allowing the NC FET to exhibit steep slope and as well increase the net gate capacitance $\left(\mathrm{C}_{\mathrm{ox}}\right)$ enabling the NC FET to deliver higher ON current in comparison to a conventional FET.
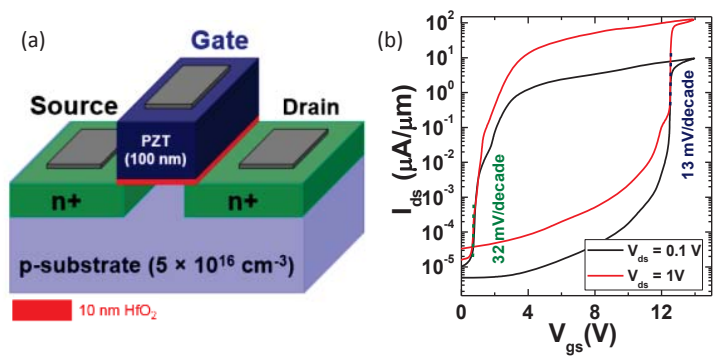

Figure 5: (a) Schematic of the NC FET showing the ferroelectric (PZT) and dielectric $\left(\mathrm{HfO}_{2}\right)$ incorporated into the transistor gate stack. (b) Experimental IDS-VGS characteristics of a PZT gated FET with channel length $=10 \mu \mathrm{m}$ for $\mathrm{V}_{\mathrm{DS}}=0.1 \mathrm{~V}$ and $\mathrm{V}_{\mathrm{DS}}=1 \mathrm{~V}$.

Figure 5(a)(b) shows the schematic and transfer (IDs- $\mathrm{V}_{\mathrm{GS}}$ ) characteristics of a NC FET developed by monolithically integrating a polycrystalline $\mathrm{PbZr}_{0.52} \mathrm{Ti}_{0.48} \mathrm{O}_{3}$ (PZT) ferroelectric with the gate dielectric of a conventional Si planar MOSFET $(10 \mathrm{~nm}$ $\mathrm{HfO}_{2}$ ). It can be observed that the transfer characteristics exhibit sub-kT/q switching (turn ON: $13 \mathrm{mV} /$ decade; turn OFF: $32 \mathrm{mV} /$ decade) owing to the $\mathrm{NC}$ in the ferroelectric [14].

The relatively large hysteresis which is a concern for voltage scaling and low voltage operation arises due to the capacitance mismatch between the ferroelectric and dielectric, and can be alleviated through scaling of the ferroelectric thickness. Additionally, scaled ferroelectrics are also necessary to maintain compatibility with the tight fin-pitch at scaled technology nodes. Scaling conventional PZT to the sub-10nm thickness has been a challenge. Thus, this has spurred the earnest development of $\mathrm{HfO}_{2-}$ $\mathrm{ZrO}_{2}$ alloys [15] which are able to exhibit ferroelectricity at scaled dimensions with appropriate materials engineering, and remains a promising direction.

\section{NON-BOOLEAN COMPUTING ARCHITECTURES}

Until now, Boolean logic has been the back-bone of information processing facilitating an excellent general purpose computing platform. However, there are certain computationally hard problems like combinatorial optimization and associative computation wherein this conventional paradigm is fundamentally inadequate. It has been proposed that dynamical systems like synchronized oscillators which incorporate inherent parallelism in their dynamics could enable an efficient solution paradigm such problems. Such systems are envisioned as analog co-processors that would augment the conventional CMOS computing platform. 
The electrically induced IMT in $\mathrm{VO}_{2}$ incorporates a fundamental instability across the phase transition. This an instability can be exploited through a negative feedback, engineered using a series resistance (e.g. MOSFET channel) to realize low-power relaxation oscillators (Fig.6(a)(b)). Further, since the computational fabric entails their coupling, a capacitive coupling scheme is used to facilitate the exchange of reactive power among the oscillators while simultaneously preventing them from disturbing each other's quiescent point [16].
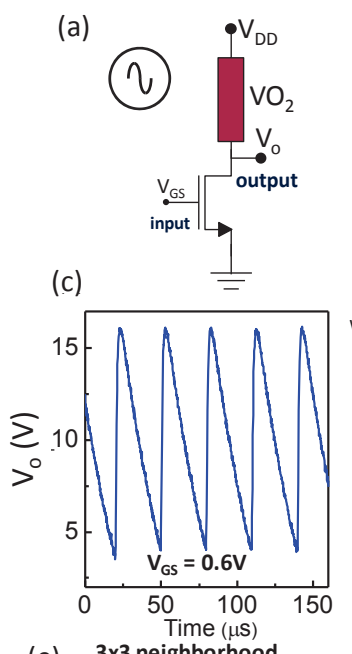

(e) $3 \times 3$ neighborhood

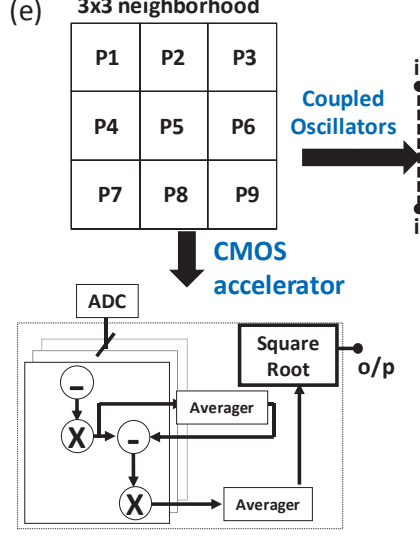

(f)

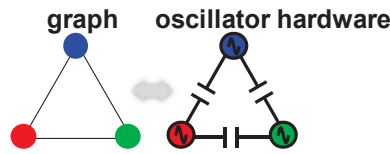

(h)

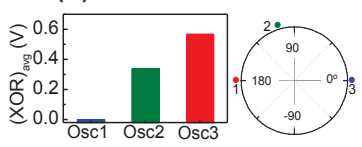

(b)

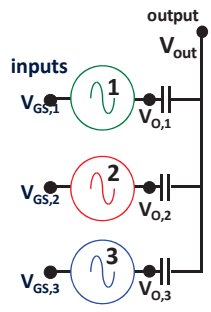

(d)

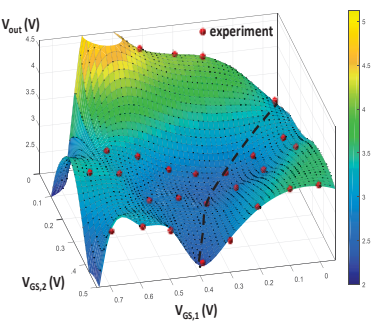

Oscillators

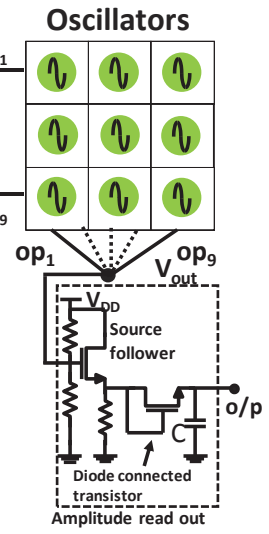

(g)

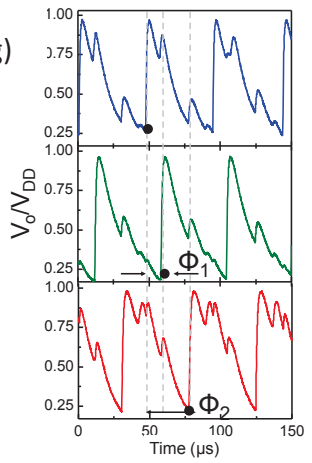

Figure 6: (a) Circuit schematic of a $\mathrm{VO}_{2}$ based relaxation oscillator. (b) Time domain waveform of the $\mathrm{VO}_{2}$ oscillator (c) Capacitively coupled $\mathrm{VO}_{2}$ oscillators (star configuration). (d) Output voltage as a function of inputs for the star coupled configuration; the attractor like characteristics enable associative computing (e) Representative schematic comparing oscillatorbased and CMOS ASIC schemes for distance computing. (f) $\mathrm{VO}_{2}$ coupled oscillators mapped to a representative graph (node $\equiv$ oscillator; edge $\equiv$ capacitor) (g) Time-domain waveform of the coupled oscillators. (h) The phase dynamics among the oscillators enable graph coloring. Phase is measured using time averaged XOR metric.

The phase synchronization dynamics of such a capacitively coupled $\mathrm{VO}_{2}$ oscillator systems provides an experimental test-bed for solving computationally hard problems. In particular, the solution to two specific problems: a) Higher order distance norm computation (for applications like Saliency detection) (Fig. $6(\mathrm{c})(\mathrm{d})(\mathrm{e}))[16][17]$ b) Vertex coloring of graphs (Fig. 6(f)(g)(h)) is experimentally demonstrated[18]. Thus, such dynamical systems could also potentially provide an alternate hardware approach to achieving computational efficiency.

\section{CONCLUSIONS}

The quest for the next generation of information processing hardware is still ongoing. Several options such as: a) transistor architectures ranging from designs that entail replacing the channel (QW FinFETs) to exploiting an alternate steep switching transport phenomena (TFETs), all the way to integrating new materials phenomena (NC FET, Phase FET); as well as b) devices and circuits that support alternate non-Boolean computing paradigms (as co-processors) are potential candidates. The eventual solution, even though currently unclear, will eventually be found through systematic and perseverant research efforts.

\section{ACKNOWLEDGMENTS}

This work was supported in part by the Center for Low Energy Systems Technology (LEAST), one of the six SRC STARnet Centers, sponsored by MARCO and DARPA. This project was also supported in part by the National Science Foundation under grant 1640081, and the Nanoelectronics Research Corporation (NERC), a wholly-owned subsidiary of the Semiconductor Research Corporation (SRC), through Extremely Energy Efficient Collective Electronics (EXCEL), an SRC-NRI Nanoelectronics Research Initiative under Research Task IDs 2698.001 and 2698.002.

\section{REFERENCES}

[1] Thompson, S.E., et al. "A logic nanotechnology featuring strained-silicon." IEEE Electron Device Letters, vol. 25, 191-193, 2004.

[2] Chau, R, et al. "Benchmarking nanotechnology for high-performance and low-power logic transistor applications." IEEE Trans. on Nanotech., vol. 4.2 153-158, 2005.

[3] Kavalieros, J., et al. "Tri-gate transistor architecture with high-k gate dielectrics, metal gates and strain engineering." VLSI Technology Symposium, Hawaii, 2005

[4] A. V. Thathachary et al, "InAs Single and Dual Quantum-Well Heterostructure FinFETs", VLSI Technology Symposium, Kyoto, 2015.

[5] A. V. Thathachary et al., "Impact of Sidewall Passivation and Channel Composition on $\mathrm{In}_{\mathrm{x}} \mathrm{Ga}_{1-\mathrm{x}}$ As FinFET Performance", IEEE Elec. Dev Lett., vol. 36 , no. 2, 117-119, 2015.

[6] A. V. Thathachary, et al., "Electron Transport in Multigate $\operatorname{In}_{x} G_{1-x}$ As Nanowire FETs: From Diffusive to Ballistic Regimes at Room Temperature", Nano Letters vol. 12, 626-33, 2014. 
[7] A. C. Seabaugh and Q. Zhang, "Low-voltage tunnel transistors for beyond CMOS logic," Proc. IEEE, 98(12), 2095-110, 2010

[8] R. Bijesh, et. al., "Demonstration of InGaAs/GaAsSb near broken-gap tunnel FET with $\mathrm{I}_{\mathrm{ON}}=740 \mu \mathrm{A} / \mu \mathrm{m}, \mathrm{g}_{\mathrm{m}}=700 \mu \mathrm{S} / \mu \mathrm{m}$ and gigahertz switching performance at $\mathrm{V}_{\mathrm{DS}}=0.5 \mathrm{~V}, " 2013$ Int. Elect. Dev. Meet. (IEDM), 28.1-.4,

[9] R. Pandey et. al., "Demonstration of p-type $\mathrm{In}_{0.7} \mathrm{Ga}_{0.3} \mathrm{As} / \mathrm{GaAs}_{0.35} \mathrm{Sb}_{0.65}$ and n-type $\mathrm{GaAs}_{0.4} \mathrm{Sb}_{0.6} / \mathrm{In}_{0.65} \mathrm{Ga}_{0.35} \mathrm{As}$ complimentary Heterojunction Vertical Tunnel FETs for Ultra-Low Power Logic," VLSI Technology Symposium,T206-T207, Kyoto, 2015

[10] Jain, A., and Alamm M. A., "Prospects of hysteresis-free abrupt switching (0 $\mathrm{mV} /$ decade) in Landau switches." IEEE Trans on Elec. Dev., vol. 60.12, 4269-4276, 2013.

[11] Madan, H., et al., "26.5 Terahertz electrically triggered RF switch on epitaxial VO2-on-Sapphire (VOS) wafer." IEEE International Electron Devices Meeting (IEDM), pp. 9-3, Washington DC, 2015

[12] N. Shukla et al., "A steep-slope transistor based on abrupt electronic phase transition", Nature Comm., vol. 6, 7812, 2015.

[13] S. Salahuddin and S. Datta. "Use of negative capacitance to provide voltage amplification for low power nanoscale devices." Nano letters, vol. 8.2, 405410, 2008.

[14] S. Dasgupta et al., "Sub-kT/q Switching in Strong Inversion in PbZr0:52Ti0:48O3 Gated Negative Capacitance FETs", IEEE J. Exploratory Solid-State Comp. Dev. and Cir., vol. 1, 43-48, 2015.

[15] Müller, J., et al. "Ferroelectricity in simple binary $\mathrm{ZrO} 2$ and $\mathrm{HfO} 2 . "$ Nano letters 12.8, 4318-4323, 2012.

[16] N. Shukla, et al., "Pairwise Coupled Hybrid Vanadium Dioxide-MOSFET (HVFET) Oscillators for Non-Boolean Associative Computing", IEEE International Electron Device Meeting (IEDM) Technical Digest, pp. 673676, San Francisco, 2014

[17] N. Shukla et al., "Ultra-low powered coupled oscillators for computer vision applications" VLSI Technology Symposium, Hawaii, 2016

[18] A. Parihar et al., "Vertex coloring of graphs via phase dynamics of coupled oscillatory networks", arXiv preprint arXiv:1609.02079 